\title{
An Integrated Floating-Electrode Electric Microgenerator
}

\author{
Wei Ma, Ruiqing Zhu, Libor Rufer, Yitshak Zohar, Fellow, ASME, and Man Wong, Senior Member, IEEE
}

\begin{abstract}
Microfabricated electric generators, scavenging ambient mechanical energy, are potential power sources for autonomous systems. Described presently are the design, modeling, and implementation of a single-wafer floating-electrode electric microgenerator, integrating a micromechanical resonator and a number of electronic devices. Forming a plate of a variable capacitor, the resonator is responsible for converting mechanical vibration to electricity. A sense transistor and a diode bridge are integrated, respectively, for monitoring the "charging" of the floating electrode and for rectification. A lumped electromechanical model of the generator is developed and expressed in terms of a set of nonlinear coupled state equations that are numerically solved. For small-amplitude excitation, a circuit based on a set of linearized equations is developed. The generator is realized using a compatible combination of standard complementary metal-oxide-semiconductor (CMOS) "floating gate" process and a post-CMOS photoresist molded electroplating process. Adequate agreement between model predictions and measurement results was obtained.

[1691]
\end{abstract}

Index Terms-Electroplating, electrostatic microelectromechanical systems (MEMS) device, energy scavenging, post-complementary metal-oxide-semiconductor (CMOS), power generator.

\section{INTRODUCTION}

$\mathbf{M}$ ICROFABRICATED systems converting vibration to electricity could be employed to scavenge ambient mechanical energy that otherwise would be wasted. Such electric microgenerators are particularly useful for powering miniaturized systems operating in poorly accessible or hostile environments. The three physical principles more popularly employed for realizing electric microgenerators are electromagnetic [1], electrostatic [2], and piezoelectric [3]. Electrostatic is a good solution for the construction of microgenerators because of its relative ease of integration with microelectronics. The technology of microfabrication enables the creation of

Manuscript received September 20, 2005; revised March 28, 2006. This work was supported by a the Institute of Integrated Micro-Systems, Hong Kong University of Science and Technology, Clear Water Bay, Kowloon, Hong Kong under a Grant. Subject Editor H. Zappe.

W. Ma is with the Department of Mechanical Engineering, Hong Kong University of Science and Technology, Clear Water Bay, Kowloon, Hong Kong, China.

R. Zhu and M. Wong are with the Department of Electrical and Electronic Engineering, Hong Kong University of Science and Technology, Clear Water Bay, Kowloon, Hong Kong, China (e-mail: eemwong@ee.ust.hk).

L. Rufer is with TIMA Laboratory, 38031 Grenoble Cedex, France.

Y. Zohar is with the Department of Aerospace and Mechanical Engineering, University of Arizona, Tucson, AZ 85721 USA.

Color versions of one or more of the figures in this paper are available online at http://ieeexplore.ieee.org.

Digital Object Identifier 10.1109/JMEMS.2006.885856 electrostatic generators employing miniaturized, capacitive electromechanical transducers.

An electrostatic electromechanical transducer consists of a variable capacitor with electrodes capable of executing a relative motion. One of the electrodes is typically implemented using a permanently charged electret. Some examples of recently implemented microgenerators are listed in Table I. All of these implementations call for the assembly of two separate substrates, one containing a mechanical resonator and another containing the electret. Such assembly requires a nontrivial alignment of the substrates that hinders scaling and optimal operation of the generators.

In this implementation, the modeling, fabrication, and characterization of a single-wafer integrated floating-electrode electrostatic electric microgenerator are described. The electret is replaced by an insulated floating-electrode made of heavily doped polycrystalline silicon (poly-Si) and fabricated using a standard CMOS "floating gate" process [7]. Charged by electron tunneling, the floating electrode works like one in a conventional nonvolatile memory device. A mechanical resonator, which forms a counter plate to the floating electrode, is realized using a photoresist molded low-temperature electroplating process [8] that is compatible with the construction of the floating electrode. A sense transistor and a diode bridge are integrated, respectively, for monitoring the "charging" of the floating electrode and for rectification.

A lumped-element electromechanical model of the microgenerator is developed and described in terms of a set of nonlinear, coupled state equations that are numerically solved using SIMULINK [9]. For small-amplitude excitation, a circuit based on a set of linearized equations is developed. The fabricated generators were tested using a shaker as a source of vibration. Reasonable agreement between model predictions and measurement results was obtained.

\section{Design AND MODELING}

Shown in Fig. 1 is the schematic design of the microgenerator. An electrically floating electrode made of heavily doped poly-Si is insulated on all sides by a low-stress silicon nitride. This electrode, together with a movable metallic electrode as the counter plate, forms a variable capacitor $C_{c f}$. It is charged by electron tunneling through a thin oxide thermally grown inside a window opened on an insulating silicon nitride. The metallic electrode functions as a laterally oscillating mechanical resonator with a loop-spring design [8]. Since significant relative displacement between the electrodes of $C_{c f}$ is possible, several cycles of variation of $C_{c f}$ between its maximum $C_{c f_{-} \text {max }}$ (fully overlapped) 
TABLE I

RECENTLY IMPLEMENTEd ElECTRET POWER MiCROGENERATORS

\begin{tabular}{|c|c|c|c|}
\hline Source & $\begin{array}{l}\text { Relative motion } \\
\text { (Capacitor gap space) }\end{array}$ & $\begin{array}{l}\text { Electret } \\
\text { (Charge density) }\end{array}$ & Remark \\
\hline Boland et al [4] & $\begin{array}{l}\text { Rotational } \\
(\sim 80 \mu \mathrm{m})\end{array}$ & $\begin{array}{l}\text { Dupont Teflon AF } \\
1601-\mathrm{S} \\
\left(-0.28 \mathrm{mC} / \mathrm{m}^{2}\right)\end{array}$ & $\begin{array}{l}\text { Frequency: } 70 \mathrm{~Hz} \\
\text { Power: } 25 \mu \mathrm{W} \\
\text { Diameter }=8 \mathrm{~mm}\end{array}$ \\
\hline Sterken et al [5] & $\begin{array}{l}\text { Lateral } \\
(2.2 \mu \mathrm{m})\end{array}$ & $\begin{array}{l}\text { SOI MPW service } \\
\text { (Not reported) }\end{array}$ & $\begin{array}{l}\text { Frequency: } 980 \mathrm{~Hz} \\
\text { Power: } 1 \mu \mathrm{W} \\
\text { Size: } 2.4 \times 1.1 \mathrm{~mm}^{2}\end{array}$ \\
\hline Arakawa et al [6] & $\begin{array}{l}\text { Lateral } \\
(300 \mu \mathrm{m})\end{array}$ & $\begin{array}{l}\text { Asahi Glass CYTOP } \\
\left(-0.68 \mathrm{mC} / \mathrm{m}^{2}\right)\end{array}$ & $\begin{array}{l}\text { Frequency: } 10 \mathrm{~Hz} \\
\text { Power: } 6 \mu \mathrm{W} \\
\text { Size: } 20 \times 20 \mathrm{~mm}^{2}\end{array}$ \\
\hline
\end{tabular}

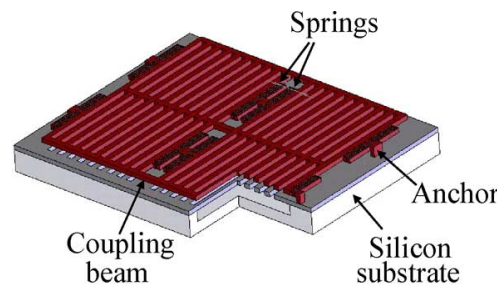

(a)

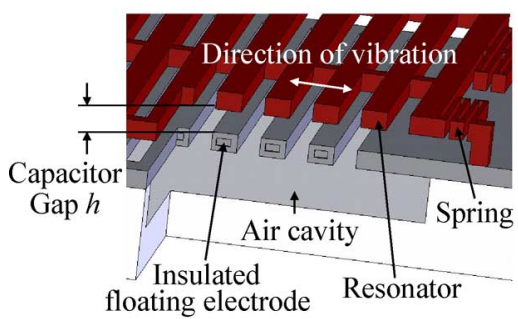

(b)

Fig. 1. Schematic diagrams of (a) a $2 \times 2$ array generator and (b) a magnified view of the resonator over the fixed floating electrode of the generator.

and minimum $C_{c f_{-} \text {min }}$ (fringe) values are possible within one vibration period.

Larger output power can be obtained by linking an array of identical resonators with rigid coupling beams [10]. This alleviates potential rigidity problems associated with a single large resonator. Furthermore, array size (hence power) can be readily scaled, while largely preserving the designed natural vibration frequency of the component resonators.

Shown in Fig. 2 is a lumped-element schematic representation of the microgenerator. It is assumed that the floating electrode is fixed to the vibration frame. The variable capacitance $C_{c f}$ is approximated by that of a one-dimensional capacitor, i.e., $C_{c f} \approx\left(\varepsilon_{o} a / h\right)(b-|x-y|)$, where $x$ is the displacement of the resonator with respect to an arbitrary but fixed origin and $y$ is the displacement of the vibration frame (hence also the floating electrode) with respect to the same origin. $\varepsilon_{o}$ is the dielectric constant of air; $h$ is the gap spacing between the electrodes; $a$ is the total effective width of the capacitor electrodes normal to the direction of motion; and $b$ is the length of the electrodes

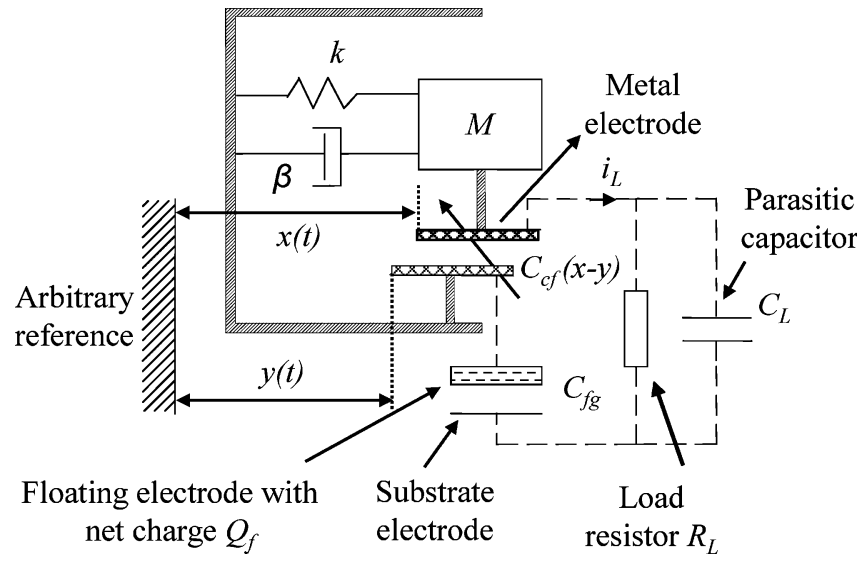

Fig. 2. Lumped-element electromechanical model of the microgenerator.

along the direction of motion. The resonator is modeled as a spring-mass system with a dashpot damper [11]. Mechanical vibration leads to changes in the relative displacement $x-y$. Consequently, $C_{c f}$ changes between $C_{c f_{-} \max } \approx \varepsilon_{o}(a b / h)$ when $|x-y|=0$ and $C_{c f_{-} \text {min }} \approx 0$ when $|x-y|=b$. Vibration leads to the generation of an alternating current flowing through an external load resistor $R_{L}$ and a parasitic capacitor $C_{L}$.

Mechanical and electrical energy conversion is coupled through the electrostatic field across the plates of $C_{c f}$. The force $F_{e \_x}$ acting on the field and the voltage $V_{e m}$ generated across $C_{c f}$ can be derived from appropriate partial differential coefficients of the total electrostatic energy $W_{e} \equiv(1 / 2)\left(Q^{2} / C_{c f}\right)$ stored in the coupling field [12]

$$
\begin{aligned}
F_{e_{-} x} & \left.\left.\equiv \frac{\partial W_{e}}{\partial x}\right|_{Q, y} \approx \frac{1}{2} \frac{Q^{2}}{C_{c f}^{2}} \frac{\partial C_{c f}}{\partial x}\right|_{y} \\
& =\operatorname{sgn}(x-y) \frac{Q^{2}}{2 C_{c f}^{2}} \frac{\varepsilon_{o} a}{h} \\
V_{e m} & \left.\equiv \frac{\partial W_{e}}{\partial Q}\right|_{x, y} \approx \frac{Q h}{\varepsilon_{o} a(b-|x-y|)}=\frac{Q}{C_{c f}}
\end{aligned}
$$

where it is assumed that there are no other energy loss mechanisms. $Q$ is the instantaneous charge on $C_{c f}$. The function $\operatorname{sgn}(x-y)=1$ if $x \geq y$ and -1 if $x<y$. 


\section{Electrical part}

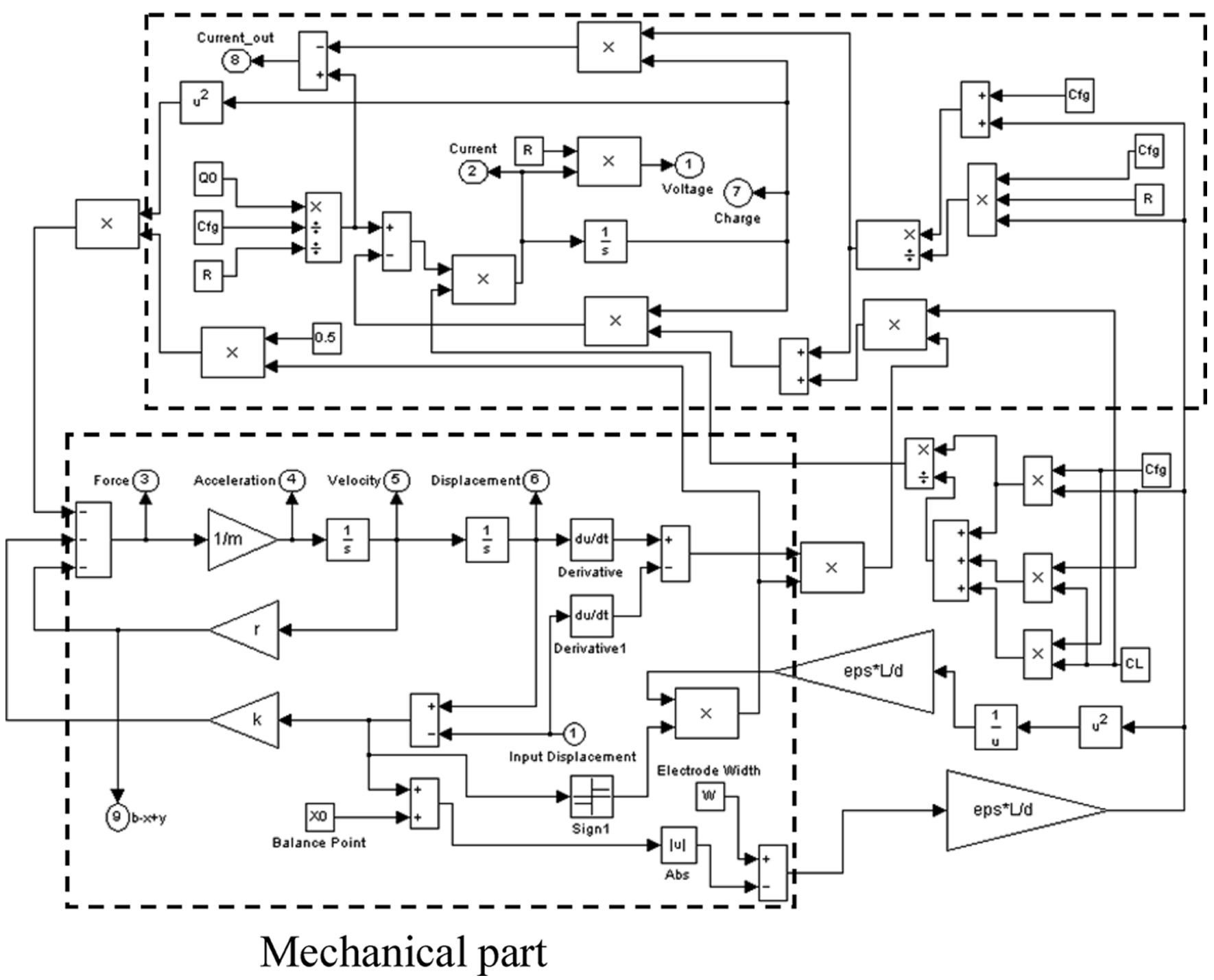

Fig. 3. Dynamic model of the microgenerator built in SIMULINK.

The mechanical resonator is governed by Newton's second law

$$
M \frac{\mathrm{d}^{2} x}{\mathrm{~d} t^{2}}+\beta \frac{\mathrm{d} x}{\mathrm{~d} t}+k(x-y)+F_{e_{-} x}=0
$$

where $M$ is the lumped mass of the resonator, $\beta$ is the damping coefficient, $k$ is the equivalent lumped spring constant, and $-F_{e_{-} x}$ is the force acting on $M$ by the coupling field.

The electrical part of the system is governed by Kirchoff's voltage law

$$
\begin{gathered}
\left(\frac{C_{c f} C_{L}+C_{f g} C_{L}+C_{f g} C_{c f}}{C_{f g} C_{c f}}\right) \frac{\mathrm{d} Q}{\mathrm{~d} t}=\frac{Q_{f}}{C_{f g}} \frac{1}{R_{L}} \\
-\left[\frac{C_{L}}{C_{c f}^{2}} \frac{\varepsilon_{o} a}{h} \operatorname{sgn}(x-y) \frac{\mathrm{d}}{\mathrm{d} t}(x-y)+\frac{C_{c f}+C_{f g}}{C_{f g} C_{c f} R_{L}}\right] Q
\end{gathered}
$$

where $C_{f g}$ is the fixed floating-electrode to ground (the substrate) capacitance, $C_{L}$ is a parasitic capacitance, and $Q_{f}$ is the net charge stored on the floating electrode. Setting $C_{L}=0$, one recovers the differential equation [13] governing the case without the parasitic capacitive loading.

It can be seen in (1) that the term $F_{e_{-} x}$ in (3) displays a quadratic dependence on $Q$ and the term $1 / C_{c f} \approx$ $\left(h / \varepsilon_{o} a\right)(1 / b-|x-y|)$ in (4) is nonlinear in $x-y$. Consequently, (3) and (4) are coupled and nonlinear.

\section{A. Large-Amplitude Solution}

Due to the difficulty in obtaining closed-form solutions to (3) and (4), a numerical approach based on a set of state differential equations is presently adopted [14]. In this approach, a given state of the system is described by a set of state variables, the time derivatives of which are determined by the present values of the state and input variables. 


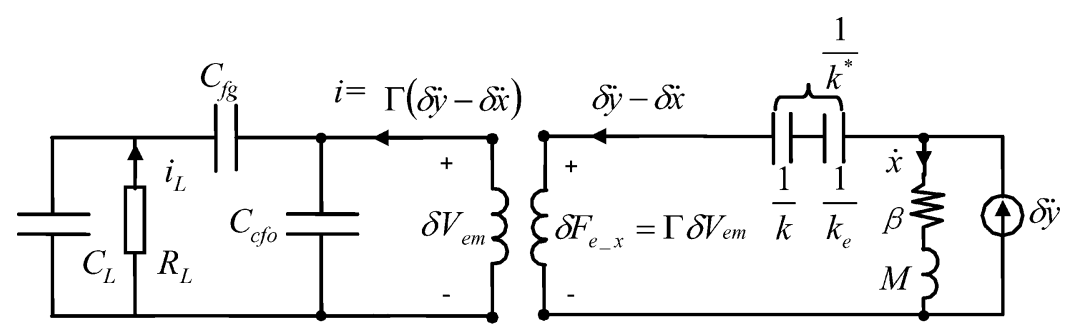

$1: \Gamma$

Fig. 4. Linear circuit network of the microgenerator.

Denoting $Q, x$, and $\mathrm{d} x / \mathrm{d} t$ by the respective state variables $X_{1}, X_{2}$, and $X_{3}$, (3) and (4) can be converted to the following set of three state equations:

$$
\begin{gathered}
\left(\frac{C_{c f} C_{L}+C_{f g} C_{L}+C_{f g} C_{c f}}{C_{f g} C_{c f}}\right) \frac{\mathrm{d} X_{1}}{\mathrm{~d} t}=\frac{Q_{f}}{C_{f g}} \frac{1}{R_{L}} \\
-\left[\frac{C_{L}}{C_{c f}^{2}} \frac{\varepsilon_{o} a}{h} \operatorname{sgn}(x-y) \frac{\mathrm{d}}{\mathrm{d} t}(x-y)+\frac{C_{c f}+C_{f g}}{C_{f g} C_{c f} R_{L}}\right] X_{1}
\end{gathered}
$$

and

$$
\begin{aligned}
& \frac{\mathrm{d} X_{2}}{\mathrm{~d} t}=X_{3} \\
& \frac{\mathrm{d} X_{3}}{\mathrm{~d} t} \approx-\frac{1}{M}\left[\beta X_{3}+k\left(X_{2}-y\right)+\operatorname{sgn}\left(X_{2}-y\right) \frac{X_{1}^{2}}{2 C_{c f}^{2}} \frac{a \varepsilon_{0}}{h}\right]
\end{aligned}
$$

where $C_{c f} \approx\left(\varepsilon_{o} a / h\right)\left(b-\left|X_{2}-y\right|\right)$, when recast in terms of the state variable $X_{2}$ and the input variable $y$. These state equations are represented in the block diagram shown in Fig. 3 and solved numerically using SIMULINK. The charge $Q$ (state variable $X_{1}$ ) is obtained as the output of the integration unit shown in the upper part of the block diagram; the displacement $x$ and the velocity $\mathrm{d} x / \mathrm{d} t$ (respective state variables $X_{2}$ and $X_{3}$ ) are evaluated in the lower part of the same diagram.

\section{B. Small-Amplitude Excitation and Linearization}

For small-amplitude input oscillation $\delta y$ about a static operating point, it is possible to obtain small-signal voltage $\delta V_{e m}$ and force $\delta F_{e_{-} x}$ by retaining only the first-order terms in the Taylor series expansions of (1) and (2)

$$
\begin{aligned}
\delta V_{e m}= & \left.\frac{\partial V_{e m}}{\partial Q}\right|_{o} \delta Q+\left.\frac{\partial V_{e m}}{\partial x}\right|_{o} \delta x+\left.\frac{\partial V_{e m}}{\partial y}\right|_{o} \delta y \\
\approx & \frac{1}{C_{o}} \delta Q+\operatorname{sgn}\left(x_{o}-y_{o}\right) \frac{Q_{o}}{C_{o}^{2}} \frac{\varepsilon_{o} a}{h} \delta x \\
& -\operatorname{sgn}\left(x_{o}-y_{o}\right) \frac{Q_{o}}{C_{o}^{2}} \frac{\varepsilon_{o} a}{h} \delta y \\
= & \frac{1}{C_{o}} \delta Q+\operatorname{sgn}\left(x_{o}-y_{o}\right) \frac{V_{o}}{b-\left|x_{o}-y_{o}\right|} \delta(x-y)
\end{aligned}
$$

$$
\begin{aligned}
\delta F_{e \_x}= & \left.\frac{\partial F_{e_{-} x}}{\partial Q}\right|_{o} \delta Q+\left.\frac{\partial F_{e \_x}}{\partial x}\right|_{o} \delta x+\left.\frac{\partial F_{e \_x}}{\partial y}\right|_{o} \delta y \\
\approx & \operatorname{sgn}\left(x_{o}-y_{o}\right)\left(\frac{Q_{o}}{b-\left|x_{o}-y_{o}\right|}\right) \frac{1}{C_{o}} \delta Q \\
& +\left(\frac{Q_{o}}{C_{o}} \frac{\varepsilon_{o} a}{h}\right) \frac{V_{o}}{b-\left|x_{o}-y_{o}\right|} \delta(x-y) \\
= & \operatorname{sgn}\left(x_{o}-y_{o}\right) \frac{Q_{o}}{b-\left|x_{o}-y_{o}\right|} \delta V_{e m}
\end{aligned}
$$

where $x_{o}$ and $y_{o}$ denote the static displacements of the resonator and the frame; $C_{o} \equiv\left(\varepsilon_{o} a / h\right)\left(b-\left|x_{o}-y_{o}\right|\right), Q_{o} \equiv$ $Q_{f}\left(C_{o} /\left(C_{o}+C_{f g}\right)\right)$, and $V_{o} \equiv Q_{o} / C_{o}$ are the respective values of the capacitance of, the charge on, and the voltage across $C_{c f}$ at the operating point. Notice that (8) and (9) do not apply when $x_{o}-y_{o}=0$, in which case $\delta(x-y)$ must be substituted by its absolute value.

For $x_{o}-y_{o}>0,(3)$ and (4) can be linearized to yield

$$
\left\{\begin{array}{c}
M \frac{\mathrm{d}^{2} \delta x}{\mathrm{~d} t^{2}}+\beta \frac{\mathrm{d} \delta x}{\mathrm{~d} t}-\left\{k+\frac{Q_{o} V_{o}}{\left[b-\left(x_{o}-y_{o}\right)\right]^{2}}\right\} \delta(x-y) \\
+\Gamma \delta V_{e m}=0 \\
R_{L}\left\{\frac{\mathrm{d} \delta Q}{\mathrm{~d} t}+C_{L} \frac{\mathrm{d}}{\mathrm{d} t}\left[\frac{\delta Q}{C_{f g}}+\frac{\delta Q+\Gamma \delta(x-y)}{C_{o}}\right]\right\} \\
\quad+\left[\frac{\delta Q}{C_{f g}}+\frac{\delta Q+\Gamma \delta(x-y)}{C_{o}}\right]=0
\end{array}\right.
$$

where the transformer factor $\Gamma \equiv \delta F_{e_{-} x} / \delta V_{e m}=Q_{o} /\left(b-\left(x_{o}-\right.\right.$ $\left.\left.y_{o}\right)\right)$. It can be seen that the coupling field behaves like a spring with a positive spring constant $k_{e} \equiv Q_{o} V_{o} /\left[b-\left(x_{o}-y_{o}\right)\right]^{2}$.

Based on (10), a linear equivalent circuit (Fig. 4) can be constructed. The electrical and the mechanical subcircuits are linked by an ideal transformer with a transformer factor $\Gamma$. The resulting linear circuit can be Fourier transformed to yield the spectrum $P(\omega)$ of the power $P$ dissipated in $R_{L}$ as shown in (11) at the bottom of the page, where $\omega_{e} \equiv 1 / R_{L}\left(C_{f g} C_{o} /\left(C_{f g}+C_{o}\right)\right)$ is the characteristic electrical angular frequency, $\omega_{m}=$ $\sqrt{\left(k^{*}+Z_{m}\right) / M}$ is the natural mechanical angular frequency, $k^{*} \equiv k+k_{e}$ is the total equivalent spring constant, $Z_{m} \equiv \Gamma^{2}\left[\left(R_{L} / /\left(1 / j \omega C_{L}\right)+\left(1 / j \omega C_{f g}\right)\right) / /\left(1 / j \omega C_{o}\right)\right]$ is the

$$
P(\omega)=\frac{\frac{\omega^{2}}{\omega_{e}^{2}} \frac{\Gamma^{2} Y^{2}}{R_{L} C_{o}^{2}}\left(\frac{\omega^{4}}{\omega_{m}^{4}}+4 \zeta^{2} \frac{\omega^{2}}{\omega_{m}^{2}}\right)}{\left(1-\frac{\omega^{2}}{\omega_{m}^{2}}-2 \zeta \frac{\omega^{2}}{\omega_{e} \omega_{m}}\right)^{2}+\frac{\omega^{2}}{\omega_{e}^{2}}\left[\left(1-\frac{\omega^{2}}{\omega_{m}^{2}}\right)\left(1+\omega_{e} R_{L} C_{L}\right)+2 \zeta \frac{\omega_{e}}{\omega_{m}}\right]^{2}}
$$




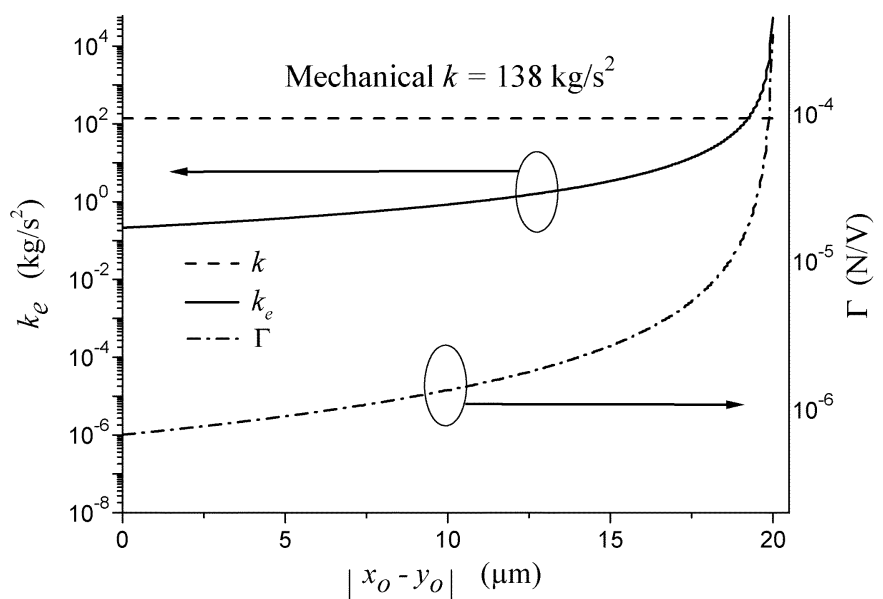

Fig. 5. Dependence of the effective spring constant $k_{e}$ and the transformer factor $\Gamma$ on $\left|x_{o}-y_{o}\right|$.

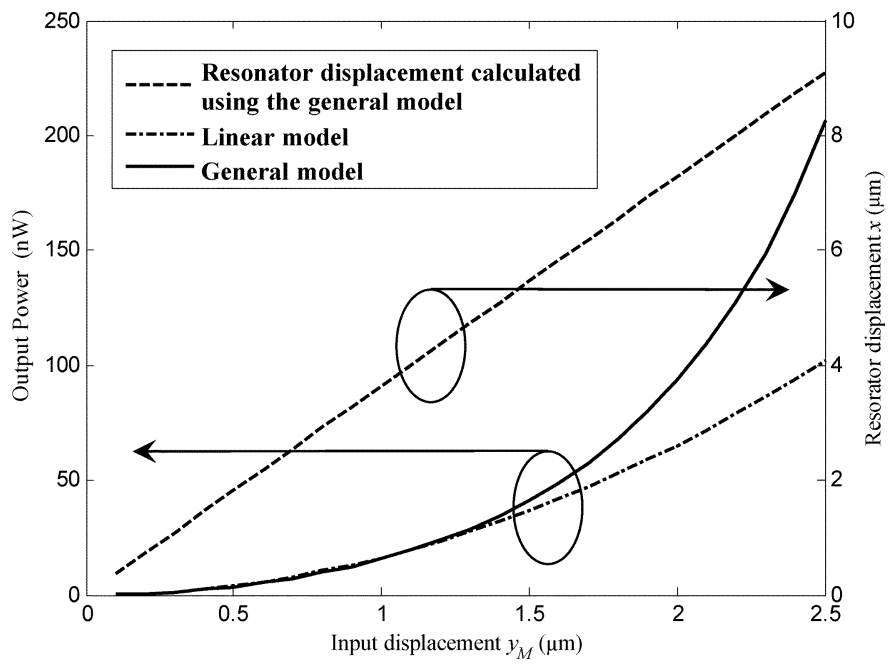

Fig. 6. Simulated dependence of the power output and the resonator displacement on $y_{M}$.

mechanical impedance induced by the electrical subcircuit, and $\zeta=\beta / 2 M \omega_{m}$ is the damping ratio of the system.

\section{SimULATION}

The values of the elements used in the models are evaluated using the appropriate material properties and the device dimensions shown in Fig. 1. The estimated values of $M$ and $k$ of the mechanical resonator are $2 \times 10^{-7} \mathrm{~kg}$ and $138 \mathrm{~kg} / \mathrm{s}^{2}$ [8], respectively. Consequently, the purely mechanical natural vibration frequency $(1 / 2 \pi) \sqrt{k / M}$ according to the linear model is $\sim 4 \mathrm{kHz}$. Based on the area of the movable capacitor plate, a value of $\sim 1 \mathrm{pF}$ for $C_{f g}$ and a value of $\sim 1.9 \mathrm{pF}$ for $C_{c f_{-} \max }$ are estimated. For a net stored charge $Q_{f}$ of $\sim 8 \mu \mathrm{C} / \mathrm{m}^{2}$, the variation of $k_{e}$ and $\Gamma$ as a function of $\left|x_{o}-y_{o}\right|$ is plotted in Fig. 5. Note that $k_{e}$ is two to three orders of magnitude smaller than the mechanical $k$ for $y_{M}<10 \mu \mathrm{m}$; hence it has negligible influence on the mechanical natural vibration frequency in this range.

With a sinusoidal driving frequency $f$ of $4.2 \mathrm{kHz}$ and amplitude $y_{M}$ ranging from 1 to $2.5 \mu \mathrm{m}, P$ dissipated on $R_{L}=$ $62 \mathrm{M} \Omega$ is obtained analytically using the set of linear model

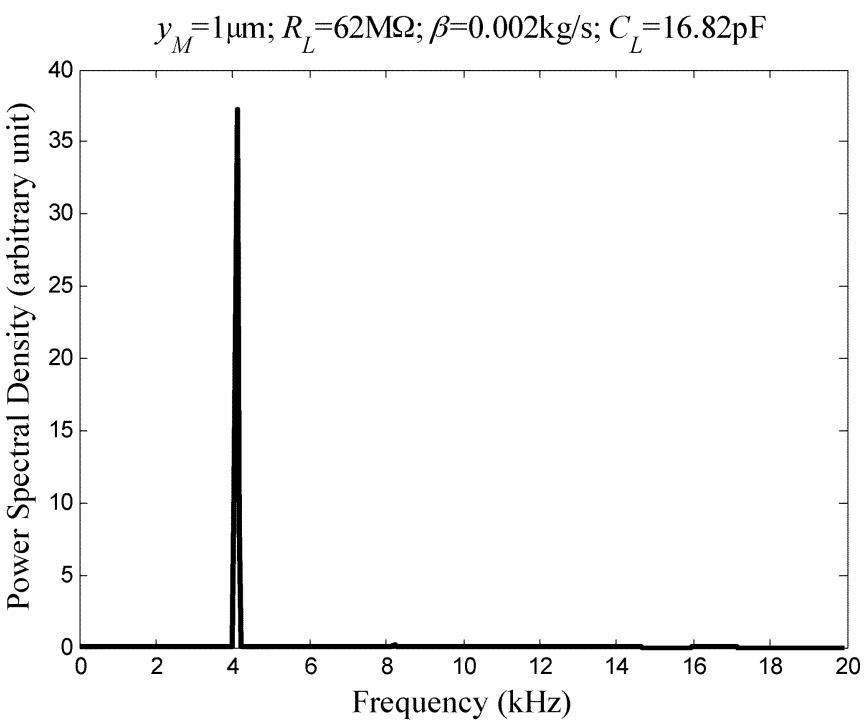

(a)

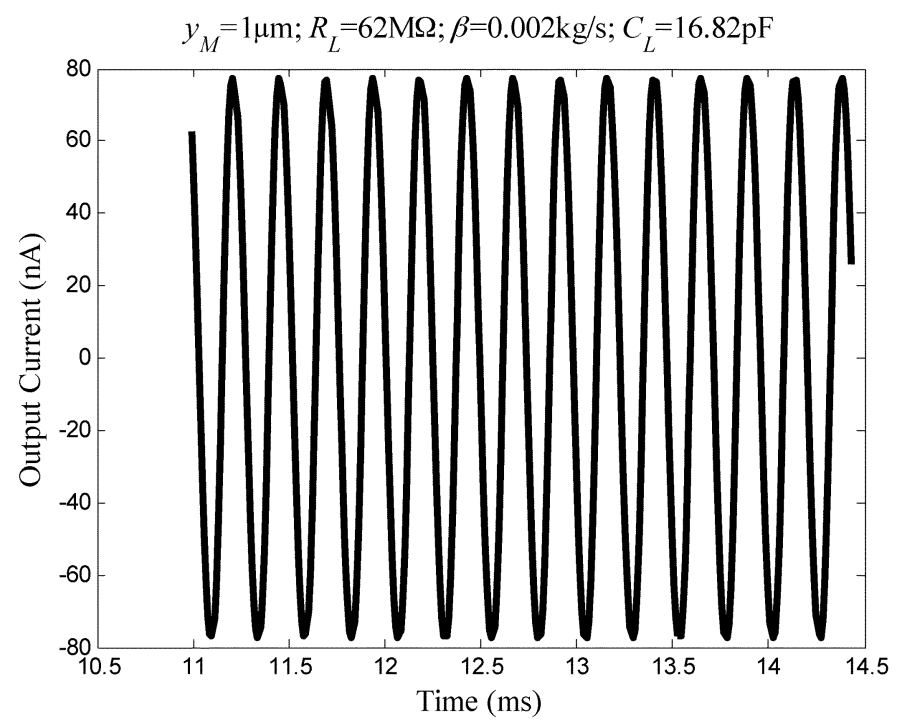

(b)

Fig. 7. (a) Frequency spectrum and (b) waveform of the current response of a $5 \times 4$ array. $y_{M}=1 \mu \mathrm{m}$.

(10) and numerically using SIMULINK for the set of nonlinear model (3) and (4). For $y_{M} \leq \sim 1.3 \mu \mathrm{m}$, the good overlap between the linear and nonlinear response shown in Fig. 6 is an indication that the device behaves essentially linearly in this range.

For $y_{M}=1 \mu \mathrm{m}$, the spectral density [Fig. 7(a)] of the waveform [Fig. 7(b)] of the current $i_{L}$ flowing across $R_{L}$ contains substantially only one component at the driving frequency, as expected for a linear system. Consequently, (11) is applied to study the dependence Fig. 8 of $P$ on $R_{L}$ and $f$ at $y_{M}=1 \mu \mathrm{m}$. Clearly, $P$ is maximum at $f \sim 4.2 \mathrm{kHz}$, close to the natural vibration frequency of $4 \mathrm{KHz} . P$ is the largest at $R_{L}=62$ and $3.2 \mathrm{M} \Omega$, respectively, for $C_{L}=0$ and $16.8 \mathrm{pF}$. The latter consists of a contribution of $\sim 14.8 \mathrm{pF}$ from the way the device is laid out and a further contribution of $\sim 2 \mathrm{pF}$ from the measurement setup.

Nonlinearity appears gradually when $y_{M}$ is increased beyond $1 \mu \mathrm{m}$. At $y_{M}=7 \mu \mathrm{m}$, an obvious distortion in the current waveform Fig. 9(a) is observed, caused by the appearance of 

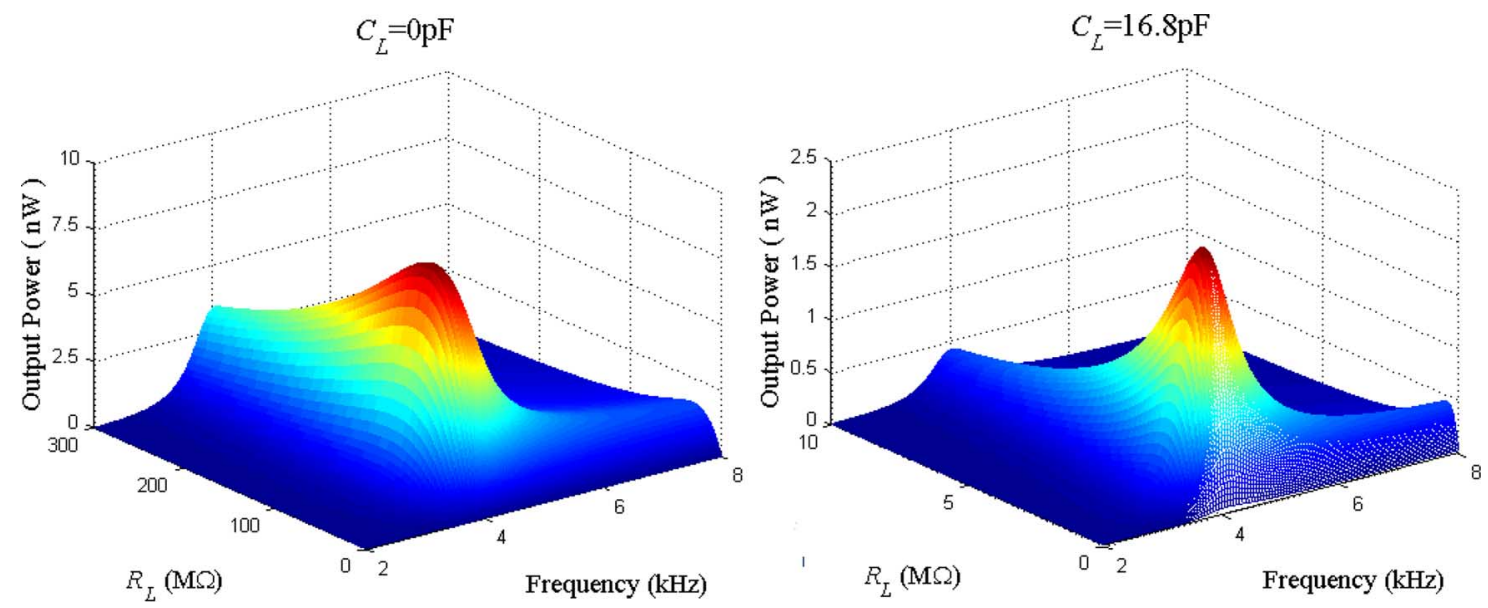

Fig. 8. Dependence of the power output of a $5 \times 4$ array on driving frequency, resistance load, and parasitic capacitance calculating using the linear model. $y_{M}=0.1 \mu \mathrm{m}$.

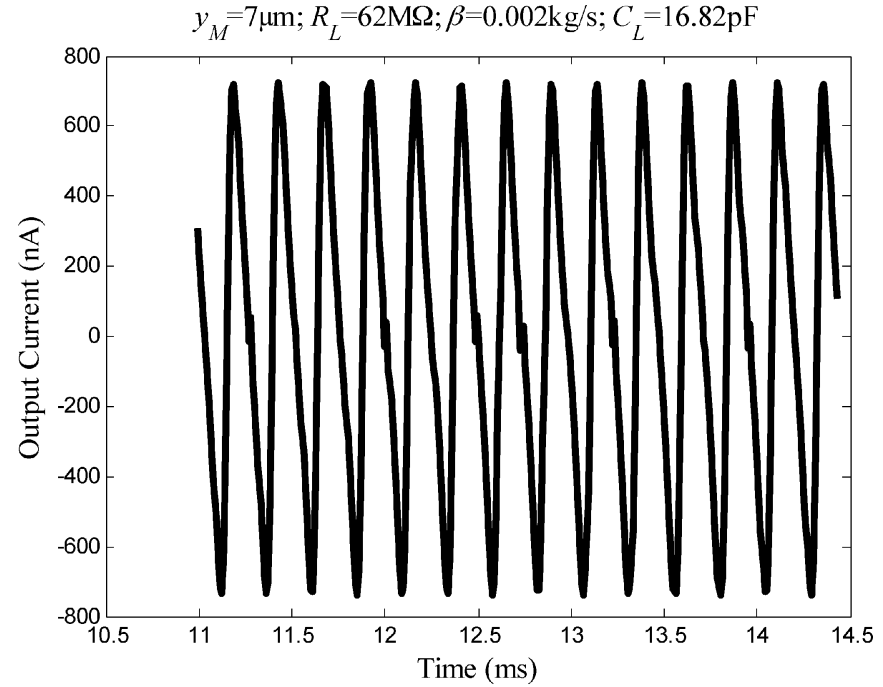

(a)

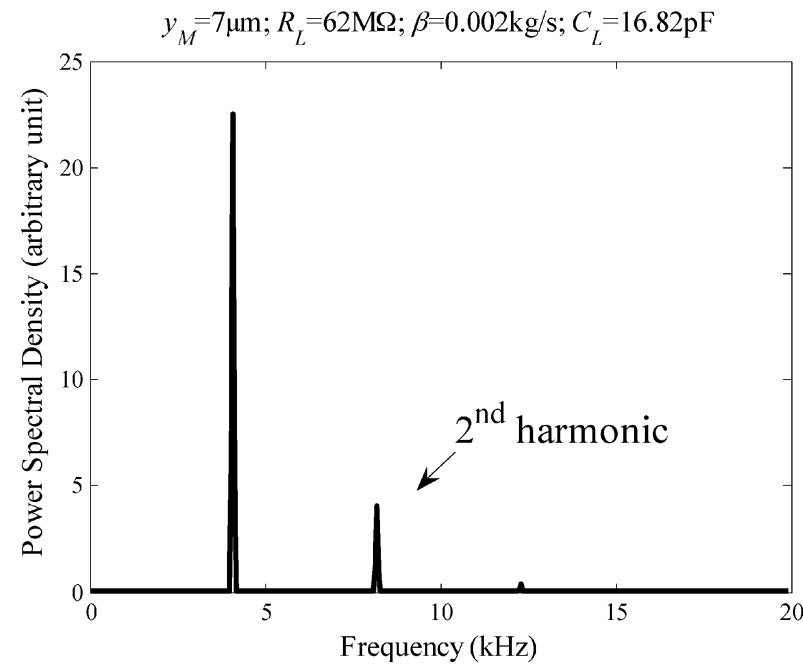

(b)

Fig. 9. (a) Waveform and (b) frequency spectrum of the current response of a $5 \times 4$ array. $y_{M}=7 \mu \mathrm{m}$.

the second- and higher order harmonics in the corresponding frequency spectrum Fig. 9(b).

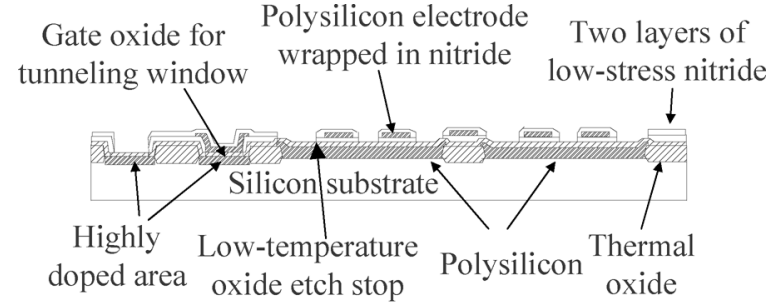

(a)

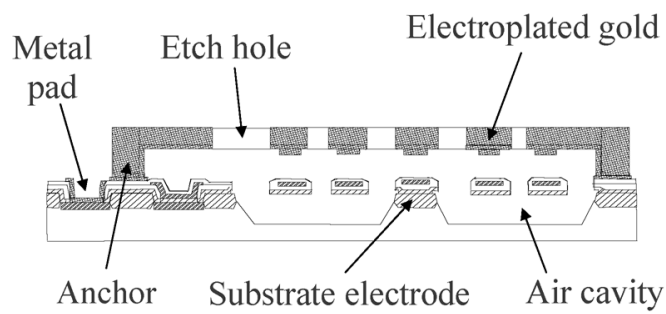

(b)

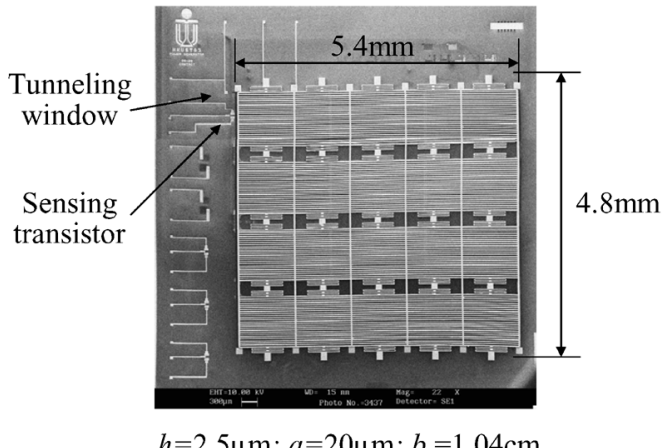

(c)

Fig. 10. Schematic cross-sections of the microgenerator after (a) the formation of the insulated floating electrode and (b) release of the resonator. (c) A secondary-electron micrograph of a fabricated $5 \times 4$ array generator.

\section{FABRICATION}

The technology Fig. 10 developed to fabricate the microgenerator consists of a conventional CMOS "front end" process to realize the floating electrode, the sense transistors, and the other electronic devices, as well as a compatible low-temperature "back end" process to realize the resonator. 


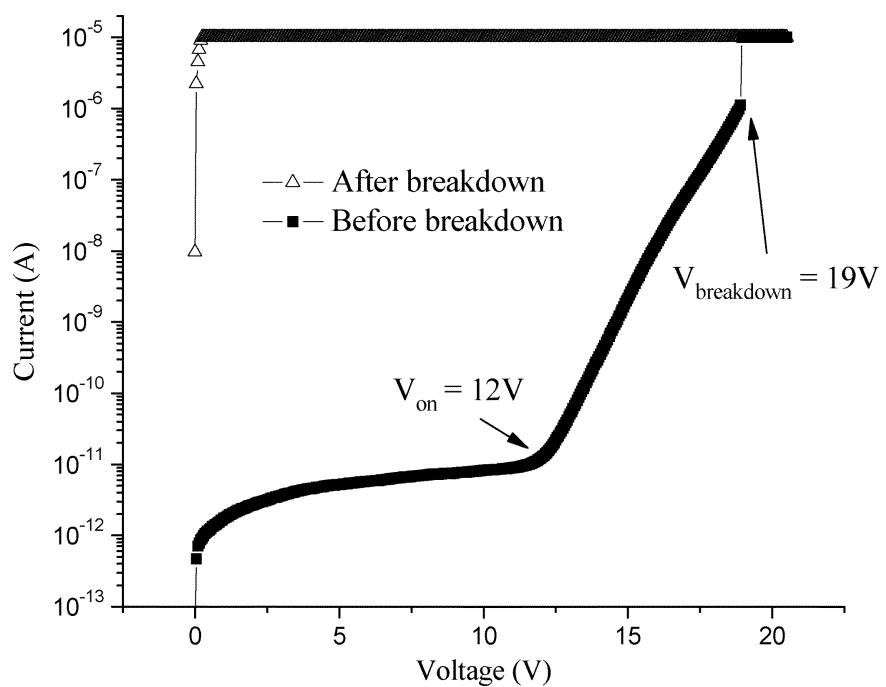

Fig. 11. Current-voltage characteristics of a tunnel oxide before and after breakdown.

The front-end process started with conventional n-type silicon substrates Fig. 10(a). The active areas were defined using a "LOCOS" isolation process, using low-pressure chemical vapor deposited (LPCVD) stoichiometric silicon nitride as the oxidation mask. A layer of sacrificial poly-Si was added to induce a subsequent large-area etching of the underlying silicon substrate to form a buried air cavity. LPCVD low-temperature oxide (LTO) was used as a diffusion mask, and heavily phosphorus-doped regions were created to form the tunneling and substrate contacts. A thin thermal oxide, $20 \mathrm{~nm}$, was formed as the "charging" tunnel oxide and the transistor gate oxide. A heavily doped poly-Si is sandwiched between two layers of LPCVD low-stress silicon nitride. This poly-Si forms the floating electrode common to both $C_{c f}$ and $C_{f g}$, as well as the gate of the sense transistor. After contact hole opening, the interconnects were realized using a double-layer of titanium/tungsten and gold ( $\mathrm{Au})$.

For the back-end resonator process [Fig. 10(b)], a first layer of $0.5 \mu \mathrm{m}$ aluminum (Al) was sputtered and patterned to form the "anti-stiction" standoff bumps. This was combined with a second layer of $2 \mu \mathrm{m} \mathrm{Al}$ to form the sacrificial layer and the conductive base layer to provide the current path needed for electroplating on the isolated seed layers. The windows on which the resonator would be anchored were opened. A $30 \mathrm{~nm} / 100 \mathrm{~nm}$ titanium:tungsten/Au adhesion/seed layer was sputtered and patterned using a liftoff technique. The resonator was then formed by Clariant AZ 4903 photoresist molded Au electroplating. Au was plated in a commercial Neutronex 309 solution at $50{ }^{\circ} \mathrm{C}$. The respective thickness of the photoresist mold and the structural Au was 40 and $36 \mu \mathrm{m}$. After the plating, all sacrificial layers were removed and the buried air cavity was formed in one immersion in a heated solution of tetramethylammonium hydroxide. A fabricated $5 \times 4$ array generator is shown in Fig. 10(c).

\section{CharaCterization}

The tunnel oxide was characterized using an HP4145B semiconductor parameter analyzer. Typical current-voltage characteristics before and after electrical breakdown are shown in Fig. 11. The respective turn-on voltage needed to initiate

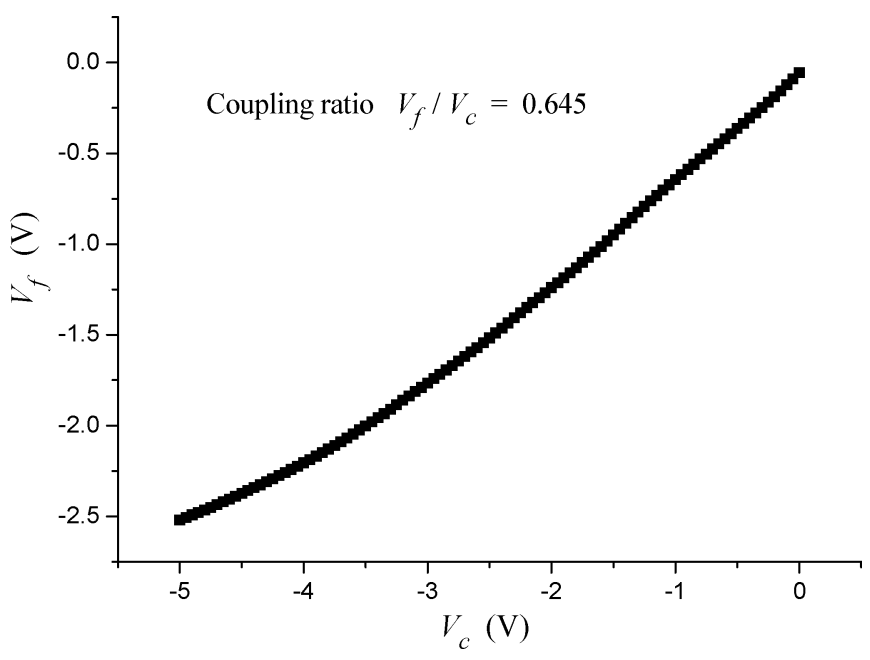

Fig. 12. Dependence of the floating electrode voltage $V_{f}$ on the control gate voltage $V_{c}$.

Fowler-Nordheim tunneling and the breakdown voltage are 12 and $19 \mathrm{~V}$, relative to an electrically grounded substrate. These define the operating range of the floating-electrode voltage $V_{f}$ at the beginning of the charging process. If $x_{o}-y_{o}=0$ during the charging process, then $V_{f}$ is related to the voltage $V_{c}$ applied on the externally accessible "control gate" (i.e., the resonator) through the "coupling ratio" $\eta \equiv C_{c f_{-} \max } /\left(C_{c f_{-} \max }+C_{f g}\right)$ and the injected charge $Q_{f}$ by

$$
V_{f}=\eta V_{c}+\frac{Q_{f}}{C_{c f_{-} \max }+C_{f g}}
$$

where $\eta$ was experimentally determined to be $\sim 0.6$ (Fig. 12). Since $Q_{f}=0$ prior to the charging process, a range between 20-30 V was determined for $V_{c}$. Furthermore, since electrons are the tunneling species during the charging process, $Q_{f}<0$ and $V_{f}$ decreases with continued charging. Charging stops when $V_{f}$ is reduced to the tunneling threshold of $\sim 12 \mathrm{~V}$.

Charging of the floating electrode was accomplished by the application of a series of voltage pulses to the control gate, with an amplitude of $25 \mathrm{~V}$ (corresponding to an initial $V_{f}$ of $\sim 15 \mathrm{~V}$ ) and a duration of $3 \mathrm{~ms}$. The charging process can be monitored by measuring the shift in the threshold voltage of the sense transistor, the gate of which is connected to the floating electrode. Since $Q_{f}<0$, a positive shift is expected. This is clearly observed in Fig. 13. If $V_{f}$ were reduced to $12 \mathrm{~V}$ such that the floating electrode were fully charged, $Q_{f}$ would be $\sim 19.2 \mu \mathrm{C} / \mathrm{m}^{2}$.

Power generation was characterized by mounting the microgenerator on an MB Dynamics Model BM 25 A shaker as a vibration source. With the shaker vibrating at an amplitude of $2.2 \mu \mathrm{m}$, the frequency dependence (Fig. 14) of the voltage waveform generated across an $R_{L}=10 \mathrm{M} \Omega$ is monitored. A maximum output power of $16 \mathrm{nW}$ is obtained with the shaker vibrating at $4.2 \mathrm{kHz}$, close to the designed mechanical natural frequency. The corresponding voltage waveform is shown in Fig. 15.

Stronger nonlinearity set in and distortion in the waveform (Fig. 16) appeared when the vibration amplitude of the shaker 


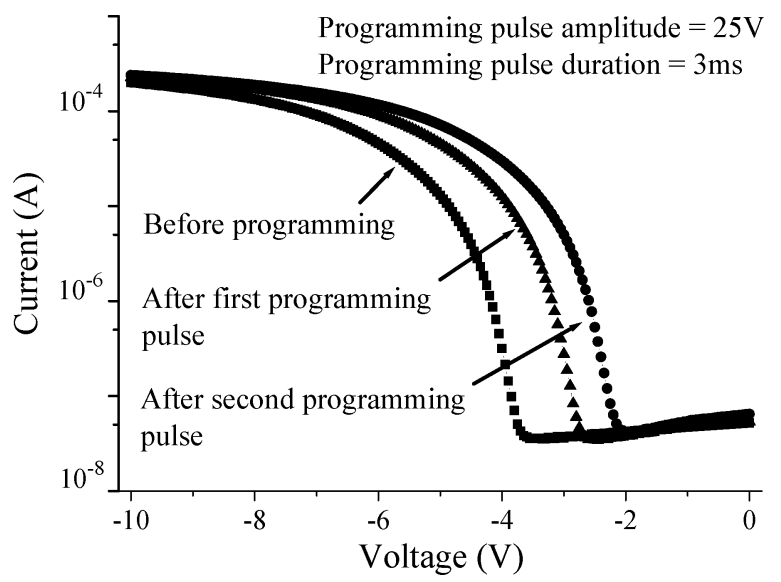

Fig. 13. Evolution of the transfer characteristics of a sensing transistor after a series of electron injection pulses.

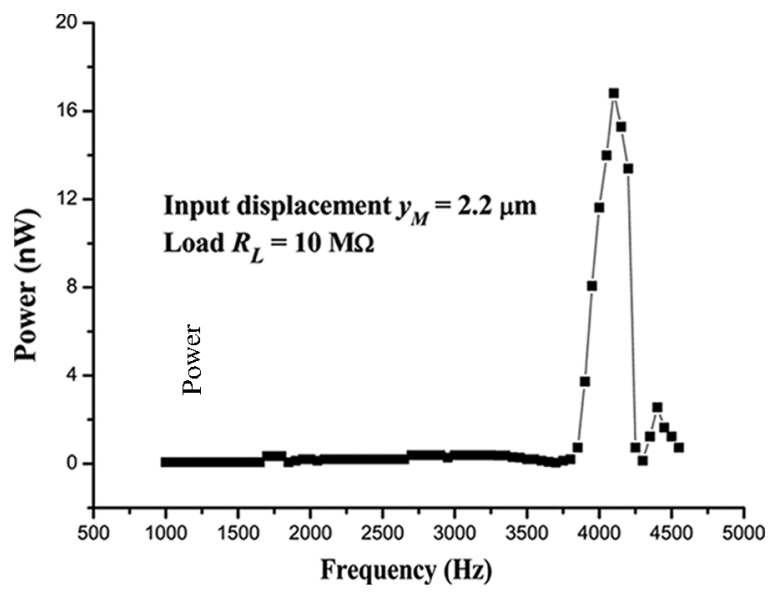

Fig. 14. Dependence of measured power output on the driving frequency.

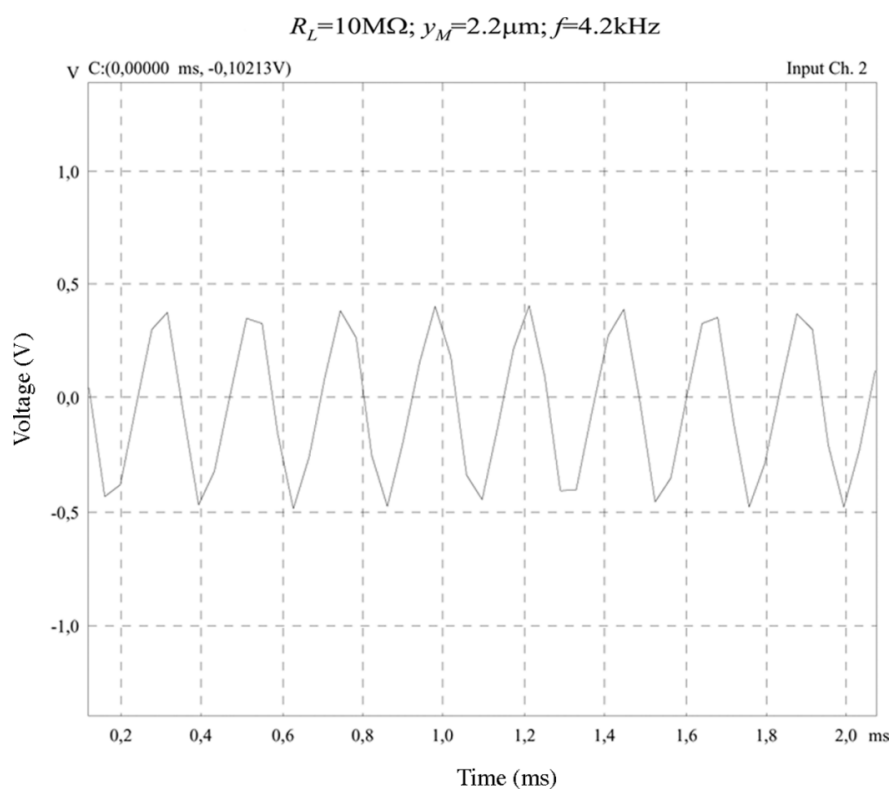

Fig. 15. Voltage waveform of a generator driven at its natural vibration frequency.

was increased to $3.2 \mu \mathrm{m}$. This distortion is a reflection of the inherent nonlinearity of the system, as described earlier.

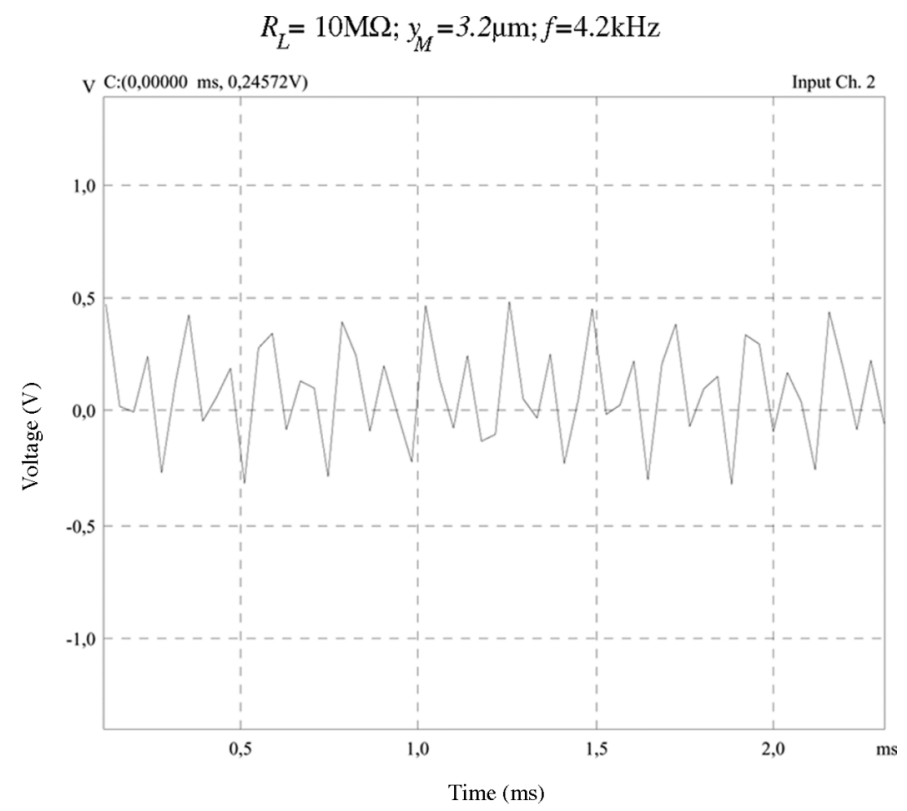

Fig. 16. Voltage waveform of a generator driven at $y_{M}=3.2 \mu \mathrm{m}$.

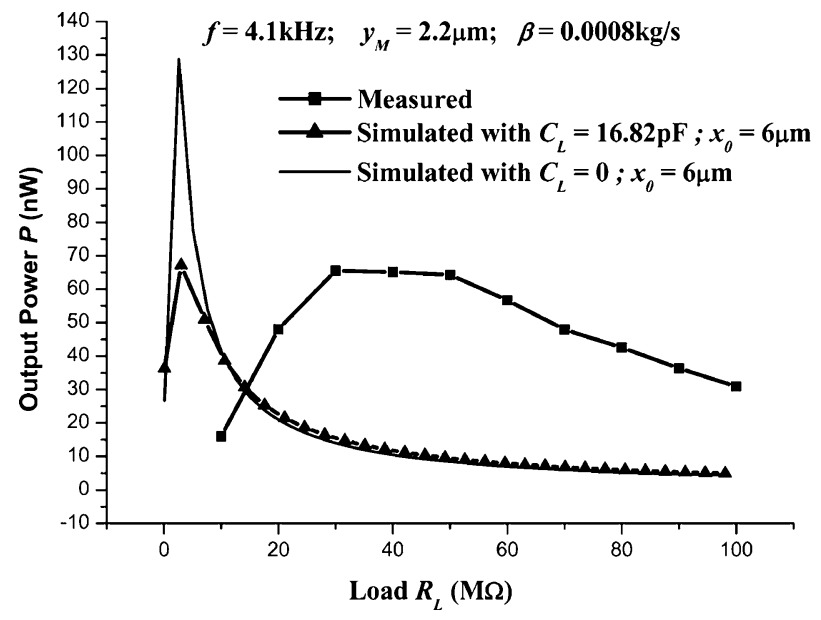

Fig. 17. Power output dependence on resistance load $R_{L}$.

With the shaker vibrating at an amplitude of $2.2 \mu \mathrm{m}$ and close to the mechanical natural vibration frequency of the microresonator, the dependence of the output power on $R_{L}$ is shown in Fig. 17. A maximum power of $65 \mathrm{nW}$ is obtained at an $R_{L}=$ $50 \mathrm{M} \Omega$, corresponding well to that simulated at the estimated $C_{L} \approx 16.8 \mathrm{pF}$. The dispersion is larger for the measured than for the simulated power output. A possible cause of such underestimation is the simplifying assumption of lumping the distributed geometry of the resonator as a "point" mass in the model equations, thus ignoring its internal dissipation and delay.

Compared to the first and third devices in Table I, the presently proposed single-wafer generator is smaller and requires no macroassembly. Compared to the second device with a microfabricated resonator and a small capacitor gap space of $2.2 \mu \mathrm{m}$, the presently proposed generator resonates at a frequency with roughly the same order of magnitude. Naturally, the actual working frequency must be tuned to each specific application. 


\section{CONCLUSION}

A single-wafer floating-electrode electrostatic electric microgenerator, integrating electronic devices for "charging" and rectification, was modeled, designed, fabricated, and characterized. Based on the electromechanical interaction through a coupling electrostatic field, a general physical model expressed in terms of a set of nonlinear coupled differential equations describing the dynamic behavior of the microgenerator was developed. For small-amplitude excitation, a circuit network based on a set of linearized equations is developed. The generator is realized using a compatible combination of standard CMOS floating-gate process and a post-CMOS photoresist molded electroplating process. Adequate agreement between model predictions and measurement results was obtained.

\section{REFERENCES}

[1] H. Kulah and K. Najafi, "An electromagnetic micro power generator for low-frequency environmental vibrations," in Proc. MEMS 2004, pp. 237-240.

[2] S. Meninger, J. O. Mur-Miranda, R. Amirtharajah, A. P. Chandrakasan, and J. Lang, "Vibration-to-electric conversion," IEEE Trans. VLSI Syst., vol. 9, no. 1, pp. 64-76, 2001.

[3] P. Gynne-Jones, S. P. Beeby, and N. W. White, "Toward a piezoelectric vibration-powered microgenerator," Proc. Inst. Elect. Eng. Sci. Meas. Technol., vol. 148, no. 2, pp. 68-72, 2001.

[4] J. Boland, Y. H. Chao, Y. Suzuki, and Y. C. Tai, "Micro electret power generator,' in Proc. MEMS'03, Kyoto, Japan, Jan. 19-23, 2003, pp. 538-541.

[5] T. Sterken, P. Fiorini, K. Baert, R. Puers, and G. Borghs, "An electretbased electrostatic $\mu$-generator," in Proc. Transducers'03, Jun. 8-12, 2003, pp. 1291-1294.

[6] Y. Arakawa, Y. Suzuki, and N. Kasagi, "Micro seismic power generator using electret polymer film," in Proc. Power MEMS 2004, Nov. 28-30, 2004, pp. 187-190.

[7] T. Ma, T. Y. Man, Y. C. Chan, Y. Zohar, and M. Wong, "Design and fabrication of an integrated programmable floating-gate microphone," in Proc. MEMS 2002, pp. 288-291.

[8] W. Ma, G. Li, Y. Zohar, and M. Wong, "Fabrication and packaging of inertia micro-switch using low-temperature photo-resist molded metal-electroplating technology," Sens. Actuators A, vol. 111, pp. 63-70, 2004.

[9] MATLAB, SIMULINK ver. 6.0.0.88, Release 12, The MathWorks, Inc., 2000.

[10] W. Ma, Y. Zohar, and M. Wong, "Integration of inertia micro-switches on active substrates containing pre-fabricated electronic devices," in Proc. APCOT-MNT 2004, Sapporo, Japan, pp. 778-782, PO2-42.

[11] W. Weaver, S. Timoshenko, and D. H. Young, Vibration Problems in Engineering, 5th ed. New York: Wiley, 1990.

[12] P. C. Krause and O. Wasynczuk, Electromechanical Motion Devices. New York: McGraw-Hill, 1989.

[13] W. Ma, Y. Zohar, and M. Wong, "Design and implementation of an integrated floating-gate electrostatic power micro-generator," in Proc. 13th Int. Conf. Solid-State Sens., Actuators Microsyst., Seoul, Korea, Jun. 5-9, 2005.

[14] S. D. Senturia, Microsystem Design. Boston, MA: Kluwer Academic, 2001.

[15] H. A. C. Tilmans, "Equivalent circuit representation of electromechanical transducer: 1-Lumped-parameter systems," J. Micromech. Microeng., vol. 6, pp. 157-176, 1996.

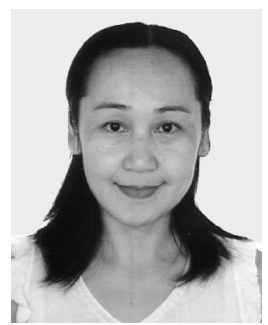

Wei Ma received the B.S. and M.S. degrees from the University of Science and Technology of China (USTC) in 1991 and 1996, respectively, and the Ph.D. degree from the Hong Kong University of Science and Technology (HKUST), China, in 2005, all in mechanical engineering. The topic of her Ph.D. dissertation was low-temperature post-CMOS microfabrication and packaging technology of MEMS devices.

She was a Lecturer with USTC from 1996 to 1997 and a Research Assistant working on microsensors and actuators with HKUST and later with Nanyang Technological University, Singapore, from 1997 to 1999. She is now a Research Scientist with the Hong Kong Applied Science and Technology Research Institute Company Ltd.

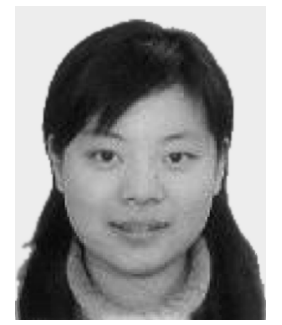

Ruiqing Zhu was born in Jiangsu, China, in 1983. She received the B.Eng. degree from the Electronics Engineering Department, Southeast University, China, in 2003. She is currently pursuing the M.Phil. degree in the Department of Electrical and Electronic Engineering, Hong Kong University of Science and Technology, China.

Her research interests are the design and fabrication of micro/nanoelectromechanical systems.

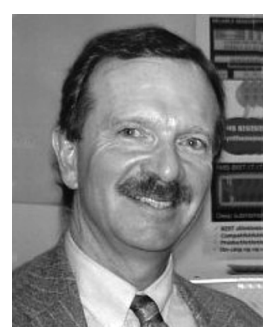

Libor Rufer received the Engineer and Ph.D. degrees from Czech Technical University, Prague, Czech Republic.

Until 1993, he was with the Faculty of Electrical Engineering, Czech Technical University. From 1994 to 2003, he was an Associate Professor with Joseph Fourier University, Grenoble, France. Since 2004, he has been with the National Polytechnic Institute, Grenoble. In 1998, he joined the Microsystems Group, TIMA Laboratory. His expertise is mainly in radio-frequency MEMS, MEMS-based sensors and actuators, electroacoustic and electromechanical transducers and their applications in acoustics and ultrasonics, associated measurement techniques, and mixed-signal systems test. Currently, he is a Member of the Reliable Mixed-Signal Systems Group, TIMA Laboratory.

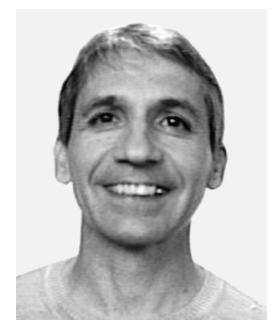

Yitshak Zohar received the B.S. and M.S. degrees in aeronautical engineering from the Technion-Israel Institute of Technology, Haifa, in 1981 and 1984, respectively, and the $\mathrm{Ph} . \mathrm{D}$. degree in aerospace engineering from the University of Southern California (USC), Los Angeles, in 1990.

As a Research Associate at USC and the University of California, Los Angeles (1990-1992), he began work on microelectromechanical systems. $\mathrm{He}$ joined the Department of Mechanical Engineering, Hong Kong University of Science and Technology, China, as one of the founding Faculty Members in 1992, where he participated in setting up the University Micro Fabrication Center and established the Department Micromachines Laboratory. At the end of 2003, he became a Professor in the Aerospace and Mechanical Engineering Department, University of Arizona, to develop a MEMS/bio-MEMS program and establish the Micro/Nano Fabrication Center. His research interests are the science and technology of microsystems, in particular microscale fluid mechanics and heat transfer. Recently, he has started to work on Bio-MEMS subjects such as micro capillary electrophoresis, patterning multi proteins, and binding kinetics of particles/cells with derivatized surfaces.

Dr. Zohar is a Fellow of the American Society of Mechanical Engineers.

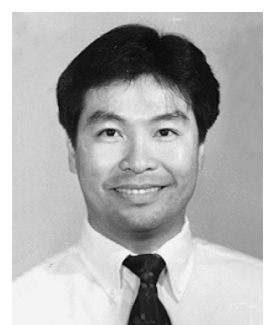

Man Wong (S'83-M'88-SM'00) was born in Beijing, China. He received the B.S. and M.S. degrees from the Massachusetts Institute of Technology, Cambridge, in 1983 and 1984, respectively, and the $\mathrm{Ph} . \mathrm{D}$. degree from Stanford University, Stanford, $\mathrm{CA}$, in 1988, all in electrical engineering.

From 1985 to 1988 , he was with the Center for Integrated Systems, Stanford University, where he worked on tungsten-gate MOS technology. From 1988 to 1992 , he was with the Semiconductor Process and Design Center, Texas Instruments, and worked on the modeling and development of integrated-circuit metallization systems and dry/vapor surface-conditioning processes. He is currently with the Department of Electrical and Electronic Engineering, Hong Kong University of Science and Technology, China. His research interests include microfabrication technology, device structure, and material; physics and technology of thin-film transistors; organic light-emitting diode display technology; and modeling and implementation of integrated microsystems.

Dr. Wong is a member of Tau Beta Pi, Eta Kappa Nu and Sigma Xi. In 2003 , he became an Honorary Guest Professor of Nankai University, Tianjin, China. 